\title{
RETAINED SURGICAL SPONGE IN ABDOMEN - A CASE REPORT
}

\section{Radiodiagnosis}

Navdeep Kaur*

Harvinder Singh Chhabra

Assistant Professor, Department of Radiodiagnosis, All India Institute of Medical Sciences, Bathinda, Punjab,India. * Corresponding Author

Senior Resident, Department of Forensic Medicine, GGS Medical College \& Hospital, Faridkot,Punjab,India.

\section{Amandeep Kaur}

Assistant Professor, Department of General Medicine, All India Institute of Medical Sciences, Bathinda, Punjab,India.

\section{ABSTRACT}

Retained foreign body is a major complication that can occur in early or delayed postoperative period having both clinical as well as medicolegal implications. We hereby report a case of 47 -year-old female with retained surgical sponge in abdominal cavity after hysterectomy. Thorough count of all sponges before and after any surgery can aid in avoiding such an undesirable event and preferable use of labelled sponges can help in early diagnosis in such cases.

\section{KEYWORDS}

Gossypiboma, Textiloma, Intestinal obstruction, Sponge

\section{INTRODUCTION}

Gossypiboma refers to a mass of cotton matrix or a sponge that is accidently left behind in a body cavity during any surgery. The risk of retained sponge is highest during emergency surgeries, in obese patients or when meticulous count of sponges is omitted after a long operating hours [1]. Use of gauze pieces without any radio-opaque marker or with damaged or disintegrated markers makes detection of any retained sponge by imaging extremely difficult. We present the case of 47-year-old female presenting with intestinal obstruction after abdominal hysterectomy with retained sponge in abdominal cavity. It posed a great diagnostic challenge due to absence of any radio-opaque marker in the sponge.

\section{CASE REPORT}

A 47-year-old Indian female presented in emergency department with complaints of severe colicky abdominal pain associated with abdominal distension and vomiting which was not relieved by medication since 1 week. She had history of abdominal hysterectomy for uterine fibroid with repair of cystorectocoele 3 weeks back. Her pain abdomen did not settle since the post-operative day and was more severe from 1 week. On examination her abdomen was distended, firm and tender on palpation. She was afebrile. Examination of other systems was non-contributory. Her hematological and biochemical parameters were within normal limits.

The patient was taken up for abdominal X-Ray for evaluation of gut obstruction which revealed a soft tissue density mass with multiple air locules trapped in it in lower abdomen. There was no evidence of any radio-opaque marker in it (Figure 1). Ultrasound abdomen showed multiple dilated gut loops along with a linear echogenic area with intense posterior acoustic shadowing in right lumbar region. This linear echogenic area was presumed to be a gas distended gut loop on initial imaging (Figure 2). Contrast enhanced abdominal CT showed a mass lesion with internal spongiform appearance with presence of few air locules in it in right lumbar region (Figure 3). The provisional diagnosis of gossypiboma was kept. Exploratory laparotomy revealed an encapsulated surgical sponge with adherent gut loops to it.

\section{DISCUSSION}

Gauze is the most common retained foreign body after any surgery and the commonest site is the abdominal cavity ${ }^{[2]}$. Gossypibomas may present at any time, within a few weeks to several decades after the initial surgery. Patients generally complain of non-specific abdominal pain, nausea, vomiting, abdominal distension, rectal bleeding, altered bowel habit, fever, anorexia, weight loss, malabsorption syndrome, or a palpable mass ${ }^{[3]}$. In the delayed phase, the presentation may be as a mass and/or intestinal obstruction.

The surgical swabs are labeled with radio-opaque markers to facilitate their detection but the markers may be damaged by repeated folding or twisting and may even gradually disintegrate with time ${ }^{[4]}$. However, surgical sponges without radio-opaque markers are still being used in many hospitals which make the diagnosis of the retained sponge by imaging extremely difficult as was seen in our case. No radio-opaque marker was seen on abdominal X-ray to suggest the possibility of a retained sponge.

Ultrasound may show a well-delineated mass containing wavy internal echoes with a hypoechoic rim and a strong posterior acoustic shadowing which changes in parallel with the direction of the ultrasound beam ${ }^{[5,6]}$. CT is the most useful modality for evaluating retained foreign bodies. CT may show a low-density lesion with infolded hyperdensities and enhancing thin or thick rim. Spongiform pattern with gas bubbles is the most characteristic CT sign of gossypiboma which was seen in our case ${ }^{[7]}$

Res ipsa loquitr, latin for "the thing speaks for itself" is the doctrine of common law. The mere presence of Gossypiboma suggests medical negligence at the part of the surgeon. A failure to reasonably diagnose the same may expose the radiologist to composite negligence where it may be alleged that the patient suffered injury/damage as a result of combined negligence of two or more doctors.

Advanced technologies such as electronic article surveillance system have been developed which electronically detects a tagged surgical sponge ${ }^{[8]}$. Bar-coded surgically sponges can be used which can be counted by bar code scanner on the table. So, the use of radiofrequency identification systems can aid in the detection of retained sponges ${ }^{[0]}$.

\section{CONCLUSION}

To conclude, the possibility of gossypiboma should be kept in the differential diagnosis of every patient presenting with any complication with positive recent or remote surgical history. Surgical swabs labeled with radio-opaque markers should be used to aid in the diagnosis of any retained sponge if any by radiographic screening after the operation.

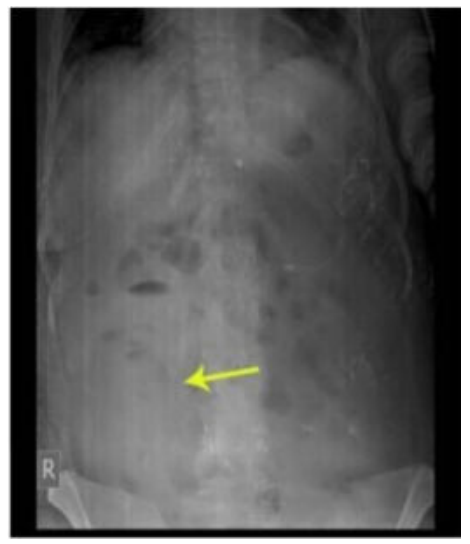



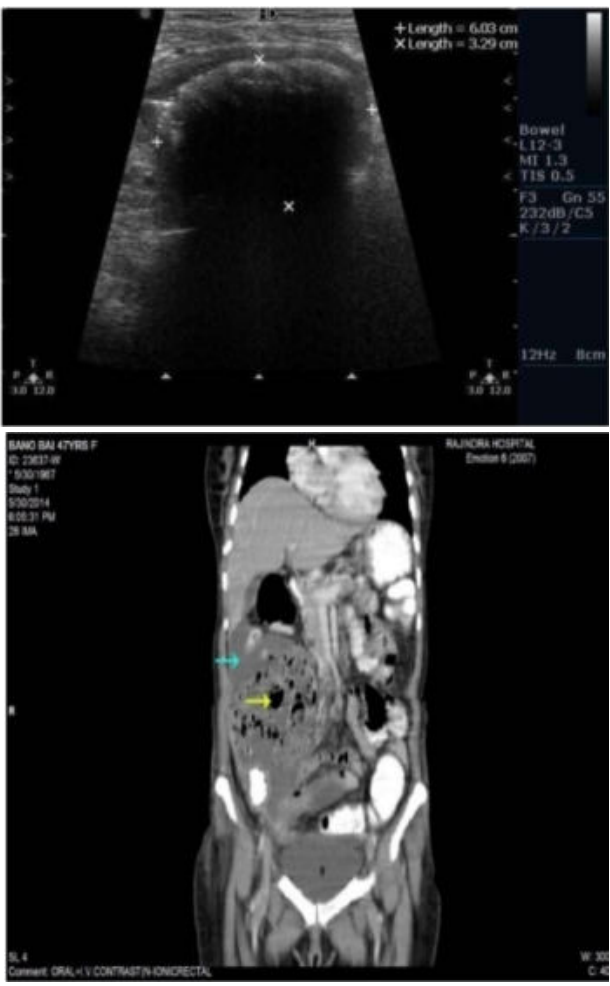

\section{REFERENCES}

Gawande AA, Studdert DM, Orav EF, Brennan TA, Zinner MJ. Risk factors for retention of instruments and sponges after surgery. N Engl J Med. $2003 \mathrm{Jan} ; 349$ (3):229-235.

Manzella A, Filho PB, Albuquerque E, Farias F, Kaercher J. Imaging of gossypibomas: pictorial review. AJR Am J Roentgenol. 2009;193: S94-101.

3. Bulus H, Sımsek G, Coskun A, Koyuncu A. Intraabdominal gossypiboma mimicking gastrointestinal stromal tumor: A case report. Turk J Gastroenterol 2011; 22:534-6.

4. Mathew RP, Suresh HB, Kaveriappa G, Bushan A. Gossypiboma presenting with the Mathew RP, Suresh HB, Kaveriappa G, Bushan A. Gossypiboma presenting with the
classical spongioform pattern. Eurorad Radiological Database 2014:1-7. classical spongioform pattern. Eurorad Radiological Database 2014;1-7.

5. Kokubo T, Itai Y, Ohtomo K, Yoshikawa K, Iio M, Atomi Y. Retained surgical sponges: CT and US appearance. Radiology. 1987; 165:415-8.

6. Chau WK, Lai KH, Lo KJ. Sonographic findings of intraabdominal foreign bodies due to retained gauze. Gastrointest Radiol. 1984;9:61-3.

7. Zappa M, Sibert A, Vullierme MP, Bertin C, Bruno O,Vilgrain V.Postoperative imaging of the peritoneum and abdominal wall.J Radiol 2009;90:969-79.

Fabian CE. Electronic tagging of surgical sponges to prevent their accidental retention. Surgery 2005; 137:298-301.

9. Gibbs VC, Coakley FD, Reines HD. Preventable errors in the operating room: retained foreign bodies after surgery. Curr Probl Surg 2007;44:281-33. 